\title{
Sistema de Informação Geográfica: uma ferramenta tecnológica de apoio de decisões aos cidadãos
}

\author{
Geographic Information System: a support tool technological for \\ citizen's decision-making
}

\author{
Claudio Luiz Chiusoli ${ }^{1}$ \\ Denis Alcides Rezende ${ }^{2}$ \\ ${ }^{1}$ Universidade Estadual do Centro-Oeste, Guarapuava, PR, Brasil \\ ${ }^{2}$ Universidade Católica do Paraná, Curitiba, PR, Brasil
}

\begin{abstract}
Resumo
No Brasil, estudos mostram que as ferramentas de sistema de informação geográfica vêm ganhando diferentes formatos de uso para tomada de decisão. O objetivo deste artigo é analisar o comportamento do cidadão em relação ao uso de informações para tomada de decisão. A metodologia da pesquisa foi caracterizada como estudo exploratório e quantitativo com amostragem não probabilística por conveniência junto a 172 cidadãos, cuja coleta foi realizada pessoalmente e por meio eletrônico. Os resultados auferidos mostram os índices de usos de mapas digitais na localização de endereços, de clientes e da concorrência, além da situação do trânsito e pontos turísticos. A conclusão do estudo reitera que $98 \%$ dos respondentes usam a ferramenta do Google Maps para localização de endereços, $76 \%$ para encontrar clientes, $73 \%$ para identificação de pontos turísticos, $65 \%$ para visualização do trânsito em tempo real e $58 \%$ para localização dos concorrentes
\end{abstract}

Palavras-chave: Sistema de Informação Geográfica. Cidade Inteligente. Geomarketing.

\begin{abstract}
In Brazil, studies show that the application of Geographic Information Systems has taken different sizes when used for decision-making. The goal is to analyze the behavior of the citizen in relation to the use of information for decision making. The research's methodology was characterized as an exploratory and quantitative study with an non-probability sampling by convenience involving 172 citizens, collected through presential and electronic channels. The results gathered show the usage percentage of digital maps for the localization of addresses, clients and business competition, as well as traffic information and touristic destinations. The conclusion of the study affirms that $98 \%$ of the subjects utilize the Google Maps research tool to locate addresses, $76 \%$ to find clients, $73 \%$ to identify touristic destinations, $65 \%$ to get real-time traffic information and $58 \%$ to locate business competition.
\end{abstract}

Keywords: Geographic Information System. Smart City. Geomarketing.

Área Tecnológica: Sistema de Informação Geográfica. Cidade Inteligente. Geomarketing. 


\section{Introdução}

A respeito do tema a ser investigado sobre sistema de informação geográfica e geomarketing no Brasil, estudos mostram a aplicação das ferramentas para apoiar na localização de lojas, no mapeamento de clientes potenciais em negócios de alimentação e na organização de equipes de venda de serviços de telecomunicação, contudo, ainda assim, poucas empresas aproveitam o potencial dessas ferramentas na gestão estratégica, que, internacionalmente, têm maior aplicação no mercado varejista (CORDEIRO, 2017).

Diante do advento da tecnologia, destaca-se que essa ferramenta tem ganhado mais espaço devido ao aumento da tecnologia de informação disponível, tornando-se uma importante aliada para que os gestores urbanos ofereçam suas cidades no âmbito digital, modelando e simulando o território para uma melhor visualização por meio de computadores, tablets e smartphones, com a proposta de melhorar a gestão da cidade com informação ao cidadão (ZHANG et al., 2016).

Assim, o uso da ferramenta, apoiado pelo geomarketing, pode ajudar o cidadão, seja ele empresário, profissional liberal, executivo, estudante ou até mesmo turista, em visita a uma localidade, nas suas decisões, tendo este se apropriado dessas ferramentas com informações da cidade em tempo real.

Nasce, assim, a importância do espaço Web para a consulta geográfica na visualização por meio de mapas digitais, a exemplo do que ocorre em outras cidades mundo afora, uma utilização na proposição para o caminho de uma cidade digital que pode ser demonstrada mediante mapas gerados com amplo apoio da internet (HIRAMATSU; ISHIDA, 2001).

Um ponto a destacar é que o sistema de informação geográfica pode ajudar no monitoramento diário dos serviços públicos e atender às necessidades dos tomadores de decisão para o acompanhamento dessas atividades mediante uma representação geográfica que, possivelmente, pode permitir redução de custos operacionais conforme o tipo de negócios que se realiza (AL-HADER, 2009). Como resultado, a informação fornecida desempenha um papel significativo na tomada de decisão, sobretudo quando a gestão pública apoia o desenvolvimento comercial e industrial com a participação dos cidadãos no exercício de suas atividades laborais, uma vez que a demanda por serviços de informações geográficas por meio da web está crescendo em todos os níveis de governo (ZHANG et al., 2014).

Tais informações de metadados da cidade, aplicadas ao estudo do sistema de informações geográfica na gestão urbana, baseado em informações sobre a cidade, se estiverem de acordo com as necessidades dos cidadãos, permitirão fornecer os mapas com estatísticas e análise dos dados pontuais (ZHU et al., 2013).

Para compreensão da ferramenta tecnológica do sistema de informação geográfica faz-se necessário estabelecer as diferenças de uma cidade digital estratégica para uma cidade inteligente, pois, muitas vezes, esses conceitos se confundem na literatura, uma vez que ambos se destacam no contexto do uso da tecnologia e têm como proposta a melhoria da qualidade de vida dos cidadãos. Diferentemente do conceito de cidade digital estratégica e de cidade inteligente, a cidade digital estratégica é compreendida como a utilização dos recursos da tecnologia da informação na gestão municipal e na disponibilização de informações e serviços aos cidadãos, assim, trata-se de um projeto mais abrangente do que apenas oferecer internet aos cidadãos por meio de recursos convencionais de telecomunicações (REZENDE, 2012). 
Estudos realizados por Dameri e Cocchia (2013), Dameri (2012; 2013), Dameri et al. (2014) e Dameri e Rosenthal-Sabroux (2014) apontam algumas diferenças entre a cidade inteligente e a cidade digital, referindo-se a um dos elementos que constituem uma cidade, a terra, que é representada pela área física. Desse modo, na cidade inteligente, a dimensão terra é o aspecto físico correspondente aos limites físicos administrativos da cidade ou região da cidade; e, na cidade digital, a terra é a representação virtual da cidade, em que as pessoas podem compartilhar dados, informação e conhecimento uns com os outros, em seus limites virtuais, em que se verificam as informações por meio dos mapas, atribuição do geomarketing.

Desse modo, a justificativa da pesquisa dá-se pelo motivo de que, hoje, cada vez mais, as cidades têm proporcionado uma mudança do ambiente físico para um mundo digital a um número crescente de cidadãos, isto é, há uma gama de disponibilização de serviços e de informações do ambiente físico para um ambiente virtual. No caso, tem-se a facilidade de obter informações por meio de mapas e de dados geolocalizados, entretanto, o ambiente físico não deixa de existir. Elas oferecem variedade de usos virtuais baseados em redes digitais de modo a observar em tempo real as atividades econômicas, políticas e culturais. Assim, as cidades digitais representam uma nova manifestação, saindo do analógico para o digital, oferecendo os mapas e os locais em relação à infraestrutura em tempo real com a presença social humana (SCHULER, 2001).

É nesse conjunto que uma cidade inteligente, a caminho de uma cidade digital, no contexto urbano, pode oferecer um bom desempenho aos cidadãos, os quais estão mais conscientes $e$ mais integrados na vida da cidade devido a um sistema de informação geográfico mais estruturado (CALDERONI et al., 2012).

Nesse contexto, apresenta-se como problema de pesquisa saber qual é o comportamento do cidadão em relação ao uso de mapas digitais, considerando a busca de informação, como: na localização de endereços, na localização de clientes e concorrentes, se observam como está o trânsito em tempo real e até se procuram pontos turísticos da cidade. De fato, utilizam os mapas digitais para localização que permite sua tomada de decisão para algum assunto de negócio ou particularidade pessoal?

O objetivo é analisar o comportamento do cidadão em relação ao uso de informações para tomada de decisão.

Como objetivos específicos têm-se como proposta analisar se é hábito utilizar: i) mapas como referência de localização na cidade, como bairros, ruas, etc. (exemplo, Google Maps); ii) mapas para localização dos clientes (exemplo, Google Maps); iii) mapas para localização dos concorrentes (exemplo, Google Maps); iv) aplicativos para visualizar o trânsito da cidade em tempo real (exemplo, Waze); e v) mapas para localização dos pontos turísticos da cidade (exemplo, Google Maps).

\section{Fundamentação Teórica}

Previamente, ao que diz respeito à fundamentação teórica, o estudo aborda os temas Sistema de Informação Geográfica (SIG) e geomarketing. Considerando esse tema, aborda-se nesta seção o sistema de informação geográfica com foco em geomarketing e sua importância para a tomada de decisão mediante o uso de mapas espaciais visualizados em formatos digitais. 
A palavra mapa teve origem na idade média, quando era empregada para a representação territorial e por meio de uma série de linhas, traços, pontos, cores e acidentes geográficos, os mapas passaram a comunicar informações e orientações desde a Pré-história (CASTROGIOVANNI, 2016).

E, assim, o geomarketing relaciona-se a quatro aspectos em suas áreas multidisciplinares, segundo apontam Cavion e Philips (2006):

a) por meio do desenvolvimento de teorias econômicas;

b) pelo aparecimento da filosofia de marketing;

c) pela colaboração dada pela geografia; $e$

d) pelo advento dos sistemas de informação geográfica.

Então, trata-se de um assunto de disciplinas múltiplas, mas seu atributo principal é o uso da tecnologia da informação com a função básica de analisar os bancos de dados com a visualização de terreno por meio de mapas espaciais, tendo a proposta de contribuir na produção, administração e análise, ajudando, assim, na tomada de decisão (HU, 2016).

Tomou a forma atual a partir de meados dos anos de 1980, com o advento dos Sistemas de Informação Geográfica (SIG) no bojo do desenvolvimento da tecnologia da informação, ao passo que, no Brasil, o geomarketing teve maior repercussão no início dos anos de 1990, sendo utilizado por empresas de franquias, bancos, shoppings e outros.

Para Latour e Le Floc'h (2001), a ideia do geomarketing refere-se a um conjunto formado por dados, sistema de informações de tratamento de dados e de métodos aplicados por um analista, elaborado para apoio à tomada de decisão sob a forma de representações espaciais ligadas à cartografia, ou seja, visualização das informações por meio de mapas.

Também conhecido como marketing geográfico, o assunto é cada vez mais usado por empresas como parte de sua inteligência de mercado. Uma ferramenta imprescindível no plano de negócios para o empreendedor ter um melhor conhecimento de seu mercado (LATOUR; LE FLOC'H, 2001). Tanto que existem várias instituições com plataforma bastante robusta em TI que oferecem serviços de mapas digitais utilizando uma larga base de dados secundários, principalmente da base do IBGE, com o apoio do SIG. Como compreensão sobre uma definição de um Sistema de Informação Geográfica (SIG), conceitua-se como um conjunto de procedimentos que fornece dados de entrada, armazenamento e recuperação, mapeamento $e$ análise espacial com atributos para apoiar as atividades da organização na tomada de decisão (VLACHOPOULOU et al., 2001).

Nesse sentido, o Sistema de Informação Geográfica (SIG) pode apoiar os cidadãos que são os gestores de empresas de diversas áreas, como logística, marketing e comercial, para avaliar opções de posicionamento de lojas, baseado em custos (transporte, mão de obra, serviços públicos, etc.), de clientes, concorrentes, entre outros atributos, conforme Vlachopoulou et al. (2001), uma vez que os dados podem ser compartilhados por vários usuários, considerando as seguranças e atualizações das informações pelos setores responsáveis, seja de ordem pública ou privada (XU et al., 2009). 
O SIG possui como características principais a possibilidade de inserir, integrar e manipular o banco de dados, provendo mapas espaciais de diversas fontes de informações, como cartografia, imagem de satélites, dados censitários e cadastro urbano e rural (FERNANDES et al., 2017).

Se a decisão exige saber a localização de clientes, os dados demográficos por agrupamento $e$, em seguida, os outros temas, como pontos de vendas, podem ser desenhados a partir dos mapas digitais com cores de identificação, pois constituem a classificação das pessoas de acordo com o tipo de bairro que vivem e com o critério socioeconômico, como renda familiar ou classe social (VEGA et al., 2015).

Dessa forma, um SIG pode utilizar a informação de atributos para apontar sua visualização por meio de mapas. Outra possibilidade é poder utilizar os logotipos de lojas e colori-los de acordo com o volume de vendas de cada unidade. Da mesma forma como pode apontar as rotas de uma atividade comercial de entrega ou coleta seletiva de algum produto ou serviço (BERNARDO; LIMA, 2017).

Em uma aplicação pontual, bairros residenciais podem ser codificados em cores por um atributo de renda média, enquanto na outra, eles podem ser coloridos com base na idade média, (HESS; RUBIN; WEST, 2003); da mesma forma, nesses serviços baseados em mapas, os cidadãos podem clicar em pontos do mapa e navegar em todos os lugares desejados (DING et al., 2003). Em resumo, a abordagem do marketing geográfico permite a compreensão de como o mercado se organiza no espaço, ou seja, permite a análise das variáveis relevantes para o marketing por meio da visualização desses dados em mapas geográficos digitais, o que antigamente era realizado manualmente, pintando ou inserindo alfinetes em mapas da cidade, bairro ou região.

Um dos fatores importantes do geomarketing é a localização, que se destaca como contexto pontual para gerar as informações, pois não basta saber qual o tamanho do mercado sem saber quais os estados, os municípios, os bairros ou as carteiras que possuem maior potencial. Sem a localização, a empresa pode deixar de identificar oportunidades de aumento de cobertura, expansão de mercado ou readequação de metas e da equipe de vendas, por exemplo (LATOUR; LE FLOC 'H, 2001). Exemplo disso é o mapeamento de clientes que preferem concentrar suas compras em lojas especializadas, e esse fluxo de compradores está trocando as tradicionais lojas de ruas por novos Shopping Centers, devido ao mix de produtos e de serviços em um mesmo ambiente (KRIŽAN, 2018).

A partir desse conceito, informações antes analisadas e visualizadas em bases de dados, planilhas e apresentações adquirem uma nova dimensão, com a consideração dos dados sociodemográficos, quando se detalha em mapas as atividades econômicas de uma determinada área (CAVION; PHILIPS, 2006).

Ainda, os autores contextualizam esse assunto fundamentando-o em três grandes disciplinas: i) a cartografia; ii) a geografia; e iii) o marketing.

O objetivo é permitir a uma empresa conhecer melhor o seu mercado, potencializando o seu desempenho por meio da equalização do mix de mercado a cada segmento delimitado geograficamente e reconhecer quais as localidades de maior potencial de consumo de um tipo de produto ou serviço (CAVION; PHILIPS, 2006). Suas principais vantagens são: i) uma melhor eficácia na tomada de decisões, possibilitando uma análise mais rápida, intuitiva e prática 
de informações complexas; ii) a otimização de investimentos; e iii) a agregação de valor nos processos de marketing direto junto ao mercado.

Conforme apontam Shao e Li (2009), em se tratando de um sistema de informação geográfica, define-se a ideia de uma arquitetura orientada para a prestação de serviço com a combinação e a utilização por meio de aplicativos executando um conjunto de tarefas, a fim de providenciar como resultado final serviços aos cidadãos, projetados em uma plataforma de partilha de informação geoespacial.

Sistemas esses que são editáveis para comunicar-se uns com os outros por esta interface, a exemplo do uso do GPS (Global Positioning System), com tradução para o português em sistema de posicionamento global, que permite aos usuários de celulares ou outros dispositivos se deslocarem, navegarem com base nas informações por meio de mapas, o que é muito comum atualmente nos celulares, por isso, a importância dos mapas digitais (MACDONALD, 2000).

Como já citado, há o exemplo do IBGE, que disponibiliza mapas digitais, atribuição ao geomarketing, de forma gratuita aos cidadãos, para acesso e obtenção de mapas personalizados por estados e município, com dados demográficos de todas as cidades brasileiras, por meio do uso do SIG, com informações pontuais com dados secundários de várias localidades. Esse sistema apresenta também a evolução da densidade no período 2000-2010 e as densidades total, rural e urbana para 2010 (mapasinterativos.ibge.gov.br/censo2010).

Dessa forma, mesmo que, às vezes, não atualizada em tempo real, torna-se de grande valia para conhecimento e auxílio na tomada de decisão. Além de uso para fins comerciais, como é o caso de um estudo de uma indústria de distribuição de automóveis que identificou os pontos fortes e os pontos fracos de uma localização para a instalação do novo negócio, mas também teve descobertas de áreas ainda não exploradas pela concorrência (DASCALU; ALBULESCU, 2013); outros estudos apontam o uso do sistema de informação geográfica para a área agrícola, que abrange dados como topografia, textura de terra, retenção e taxa de infiltração de água na terra e sistema de drenagem de campo para pomares de mirtilo (HOCES et al., 2012).

Esses mapas visualizados por meio da ferramenta de TI e pelo Sistema de Informação Geográfica (SIG) permitem obter muitas informações de grande utilidade e sem custo, porém ainda são pouco conhecidos e utilizados pelos cidadãos, mas com grande potencial de se tornarem uma tecnologia chave para cidades digitais (ISHIDA, 2000).

Ao contrário de mapas em papel, o SIG é capaz de armazenar, manipular e exibir um conjunto muito mais rico de informações de atributo, visto que é um sistema de gerenciamento de banco de dados especializado que aponta conjuntos de características e atributos de um negócio qualquer (HESS; RUBIN; WEST, 2003).

Também é possível obter vários mapas a partir de serviços oferecidos por empresas que trabalham com a proposta de inteligência de mercado a partir de uma ampla base de dados secundários, relacionando a população em seus diversos graus de informação, com fontes secundárias do IBGE, como números de bancos, escolas, franquias, entre muitas outras variáveis. Essas informações são disponibilizadas por meio de mensalidades ou pagamentos de estudos encomendados.

Além disso, é possível que o assinante crie seus próprios mapas por meio de sua base de dados, como cadastro de compradores atuais, ou também gerando por meio de pesquisas para posteriormente mapeá-los. Há algumas ferramentas disponíveis que permitem o uso de 
um sistema de geomarketing para mapear as informações utilizando o SIG, que diz respeito ao Batchgeo (2017) (www.batchgeo.com), que permite o mapeamento de endereços e dados de clientes, fornecedores, concorrentes, entre outros.

Considerando o exposto, reitera-se a importância da informação para a tomada de decisão quando visualizadas por meio dos mapas digitais e dados estatísticos ofertados. Assim, entende-se como ponto de partida a inclusão digital, que é o acesso à informação por meios digitais; $e$ o ponto de chegada, a assimilação da informação e sua reelaboração em novo conhecimento auxiliando a tomada de decisão (SIMÃO, 2010).

Nesse sentido, o SIG é um grande auxiliador para a construção de uma cidade digital, porque envolve aplicação integrada de modernas tecnologias, mapas de informação espacial, serviços baseados em localização, até para grandes projetos imobiliários em que se precisa de um plano diretor por meio de banco de imagens e informações (AL-HADER, 2009). Uma vez que em 1960 o uso de SIG em planejamento urbano tornou-se muito comum na América do Norte, Europa Ocidental, Japão e Austrália devido à sua aplicação no uso do solo, zoneamento, análise do local para a sustentabilidade e desenvolvimento econômico.

Agora, o SIG também está nos países em desenvolvimento, como a Índia, China, México, Brasil, África do Sul, Gana e Senegal, mostrando ser uma excelente tecnologia que permite aos planejadores urbanos integrarem uma grande variedade de dados de múltiplas fontes, com capacidade de entrada, armazenamento, manipulação, análise e visualização espacial (KUMAR, 2016).

A informação espacial forma o conhecimento do território e auxilia nos movimentos humanos dentro de seu ambiente, por meio do conhecimento espacial sensorial e comportamental; assim, uma cidade com a proposta de se tornar digital, apoiada pela internet, oferece aos cidadãos todos os tipos de informações em tempo real, bem como possibilidades de comunicação e interação.

Dessa forma, o espaço é uma infraestrutura de informação social para o cotidiano urbano, enviando e recebendo informações e comunicação interativa (CHANG, 2003).

Nesse caso, as características mais importantes do SIG são: i) a facilidade de integração e entrada mediante várias fontes de dados; ii) a manutenção consistente dos dados; iii) a facilidade de atualização dos dados; e iv) a flexibilidade no armazenamento e recuperação de dados. E devido às características mencionadas, o sistema de informação geográfica encontra um lugar importante no planejamento urbano e é útil como uma ferramenta muito poderosa para o planejamento e gestão urbana (KUMAR, 2016).

O sistema de informação geográfica aliado à realidade virtual permite a modelagem $e$ a simulação de uma cidade visualizada no computador e, portanto, permite uma melhor gestão de uma cidade. Modelar e simular a cidade em uma escala tridimensional com ligação com a geometria espacial tornou-se um tema recorrente entre os especialistas das áreas de TI, cuja plataforma integra as tecnologias de sistema de informação geográfica, realidade virtual e banco de dados, podendo desempenhar o papel de base para uma cidade digital sobre o qual outros sistemas de aplicação são unidos (ZHANG et al., 2016).

Pensando nessa dimensão espacial, Ishida (2017) reforça como projeto para uma cidade digital um espaço Web com uma linguagem que apoia a consulta geográfica e, consequentemente, a criação do plano de utilização em uma cidade digital, baseada na cidade demonstrada, por 
meio de mapas pela internet. Esse espaço Web envolve o conceito da abordagem em integrar a world wide web (www) e um Sistema de Informação Geográfica (SIG), que consiste em home pages e hiperlinks representando as relações geográficas entre elas.

A conotação da informação geográfica tem se enriquecido pelo desenvolvimento de uma grande variedade de aplicativos, os quais são nos formatos tridimensionais, ao invés de modelo tradicional de vetor. Assim, a programação de 3D fornece um cenário de uma cidade mais realista aos cidadãos com as coordenadas geográficas (W-Q MAO, 2014).

Esses cenários mais realistas criam uma dinâmica de utilizar melhor as funções de avaliação geográficas; a exemplo, uma consulta de pesquisa na qual um cidadão usuário deseja saber onde está e o caminho que deseja ir, como ferramenta para diversas ocasiões, seja a negócio, estudo ou lazer. Logo, a dimensão de uma boa governança municipal com uso de mapas digitais em cidades inteligentes torna-se uma forma aberta e democrática de uso para os cidadãos, com benefício dos recentes avanços no sistema de informação geográfica, pautado em três frentes, como reitera Roche (2014): i) a cooperação com a transversalidade e a parceria; ii) a participação da construção de políticas públicas mediante consultas e debates; e, por fim, iii) um portal da transparência com dados abertos para demonstrar e esclarecer o uso de imagens.

\section{Metodologia da Pesquisa}

Como método de pesquisa, trata-se de um estudo exploratório mediante revisão da literatura em artigos nacionais e, principalmente, internacionais, destacando-se com descobertas de ideias e intuições, cujo objetivo é familiarizar-se e conseguir nova compreensão sobre o assunto para poder formular um problema de pesquisa mais adequado e criar os testes de hipóteses (GIL, 2008; MARCONI; LAKATOS, 2010; CERVO; BERVIAN, 2007).

Ainda houve um survey, mediante a coleta de dados que em relação ao objetivo pode ser considerado um estudo descritivo, uma que vez que pretendeu analisar o comportamentos, hábitos e atitudes dos cidadãos relacionados ao tema estudado (ROESCH, 1999; SIEGEL; CASTELLAN JR., 2017; MATTAR, 2000; MALHOTRA, 2001; FREITAS et al., 2000).

Foi utilizado um estudo quantitativo sobre a natureza das variáveis, nesse estudo, como foi citado por Malhotra (2001), os dados são quantificados para aplicação dos testes estatísticos propostos na metodologia.

Quanto ao tempo, foi compreendido por um recorte transversal, uma vez que a coleta dos dados ocorreu somente uma única vez, pretendendo descrever e analisar o estado das variáveis nesse dado momento (FREITAS et al., 2000).

Em relação à técnica de amostragem, foi utilizada a não probabilística por conveniência com 172 cidadãos; uma vez que não foi realizado sorteio para as entrevistas, pois foi utilizada como unidade de observação direcionada para empresários, profissional liberal, autônomo e executivos em uma cidade do estado do Paraná, como parte integrante do estudo. Nesse caso, partiu-se da premissa que eles utilizam as ferramentas de SIG e geomarketing para entender e compor suas estratégias de mercado, tomada de decisão e alguns casos, para uso pessoal.

O questionário foi estruturado em duas formas, utilizando-se da tecnologia do Google Forms, e um segundo questionário para coleta dos dados por meio de entrevistas pessoais, cujo período da coleta foi no ano de 2017. 
O modelo que instrumentou a coleta de dados consta de cinco variáveis, nas quais houve aplicação de 10 pré-testes para uma melhor configuração para dar validade e confiabilidade à pesquisa, cujo objetivo era refinar o questionário, com intuito de garantir que realmente foi medido aquilo a que se propôs (FREITAS et al., 2000; MARTINS, 2006).

Após elaborada e aplicada a coleta de dados, chegou-se ao seguinte perfil de entrevistados: i) predominância dos homens (70\%), contra $30 \%$ da participação das mulheres; ii) maior concentração de entrevistados entre 31 a 50 anos (quase 60\%); e iii) nove a cada 10 respondentes $(90 \%)$ têm acesso à rede social e a usa. Dessa forma, para as análises mediante cruzamentos de dados e teste de hipótese, houve um agrupamento entre as variáveis gênero e faixa etária na seguinte configuração: gênero com idade até 40 anos e acima de 40 anos.

Frisa-se que para aplicação dos testes e análise dos resultados, a escala utilizada, que era "sempre uso", "às vezes", "raramente" e "não uso", foi agrupada para fins de estatística descritiva e aplicação do teste Qui-Quadrado como "uso" e "não uso", para dar força à aplicação do teste estatístico. Já para o teste de correlação de Spearman, foram mantidas originalmente como aplicadas, uma vez que se trata de uma escala ordinal.

A partir disso, elaborou-se o protocolo de pesquisa para atender aos objetivos e à questão-problema, considerando seus constructos em relação à fundamentação teórica, às variáveis e às questões correspondentes, suas escalas e técnicas de coletas. Assim, além do perfil dos entrevistados os constructos (cinco variáveis) sobre sistema de informação geográfica e geomarketing foram a respeito do uso de: 1) mapas para localização de bairros e ruas (var1); 2) mapas para localização dos clientes (var2); 3) mapas para localização dos concorrentes (var3); 4) aplicativos para visualização do trânsito (var4); e 5) mapas para localização dos pontos turísticos (var5).

Após a obtenção dos dados coletados, foram armazenados e processados eletronicamente no programa SPSS (v.22). A análise dos dados consistiu em análises univariadas e bivariadas com frequências absolutas e relativas. As medidas de associação foram testadas por meio do teste não paramétrico, o Qui-Quadrado e o teste de correlação de Spearman (SIEGEL; CASTELLAN JR., 2017).

Para o teste de correlação de Spearman, valendo-se que o p (positivo ou negativo) pode variar entre correlação bem fraca (até 0,19$)$, correlação fraca $(0,20$ a 0,39$)$, correlação moderada $(0,40$ a 0,69$)$, correlação forte $(0,70$ a 0,89$)$ e correlação muito forte $(0,90$ a 1$)$ para medir a intensidade da relação entre variáveis ordinais. Todos os resultados das análises bivariadas foram avaliados com nível de significância estatística de 5\% ( $p<0,05)$.

Desse modo, postula-se as hipóteses da pesquisa, a partir dos conceitos sobre Sistema de Informação Geográfica (SIG) e geomarketing e, assim, as medidas de associação foram testadas por meio do teste não paramétrico, o Qui-Quadrado e correlação de Spearman, cujas hipóteses da pesquisa são:

Quadro 1 - Testes de hipóteses

\begin{tabular}{|c|c|}
\hline Teste não paramétrico Qui-Quadrado & $\begin{array}{r}\text { H1: Não há diferença significativa no comportamento dos } \\
\text { cidadãos quanto ao uso de mapas virtuais considerando } \\
\text { os grupos pesquisados: gênero e faixa etária. }\end{array}$ \\
\hline $\begin{array}{c}\text { Teste não paramétrico de } \\
\text { correlação de Spearman }\end{array}$ & H2: Há forte correlação positiva entre as cinco variáveis pesquisadas \\
\hline
\end{tabular}

Fonte: Elaborado pelos autores deste artigo (2017) 


\section{Resultados e Discussão}

Considerando esta parte do estudo, são indicados os principais achados mediante estatística descritiva. Posteriormente com a análise do teste não paramétrico: Qui-Quadrado e correlação de Spearman para validar o teste de hipótese se deve rejeitar ou não rejeitar.

\subsection{Análise da Estatística Descritiva}

A partir da estatística descritiva e do perfil já mencionados na metodologia, chegou-se aos índices de uso das informações em relação as cinco variáveis investigadas. Verifica-se, assim, por meio do Quadro 2, que o uso de mapas como ferramenta a exemplo do Google Maps, de forma geral, é bastante intenso como referência na localização de endereços da cidade, bairros e ruas (var1), com 97,7\% de citação.

Em destaque, na sequência, aparece o uso de mapas para localizar os clientes (var2), com $75,6 \%$, cujo indicador é maior entre os cidadãos de 31 a 50 anos (84,9\%). Em seguida, a localização dos pontos turísticos da cidade (var5) aparece com 72,7\%, cujo índice é maior entre as mulheres (82,7\%), principalmente com idade acima de 40 anos (83,9\%).

$\mathrm{O}$ que foi menos utilizado entre os pesquisados refere-se à visualização do trânsito da cidade em tempo real (var4), com 64,5\%, sendo que esse indicador é menor entre os cidadãos com faixa etária de 41 a 50 anos (53,3\%). Quanto ao uso de mapas para localização dos concorrentes (var3), aparece com 58,1\%, com índice menor entre os homens acima dos 40 anos $(47,1 \%)$. Aqui, cabe um comentário adicional: os cruzamentos das variáveis por gênero e faixa etária apontam um razoável índice de mapeamento de concorrentes e isso sugere que seja mais elevado para perfis de respondentes que se enquadram em um perfil de empresário, executivo ou profissional liberal.

Quadro 2 - Estatística descritiva sobre o uso do sistema de informação geográfica e geomarketing

\begin{tabular}{|c|c|c|c|c|}
\hline & VARIÁveIs - Uso DE: & Uso & NÃo Uso & TotaL \\
\hline Var1 & $\begin{array}{c}\text { Mapas como referência de localização da cidade, } \\
\text { como bairros, ruas, etc. (exemplo, Google Maps) }\end{array}$ & 97,7 & 2,3 & 100 \\
\hline Var2 & Mapas para localização dos clientes (exemplo, Google Maps) & 75,6 & 24,4 & 100 \\
\hline Var3 & Mapas para localização dos concorrentes (exemplo, Google Maps) & 58,1 & 41,9 & 100 \\
\hline Var4 & $\begin{array}{c}\text { Aplicativos para visualizar o trânsito da cidade } \\
\text { em tempo real (exemplo, Waze) }\end{array}$ & 64,5 & 35,5 & 100 \\
\hline Var5 & $\begin{array}{c}\text { Mapas para localização dos pontos turísticos } \\
\text { da cidade (exemplo, Google Maps) }\end{array}$ & 72,7 & 27,3 & 100 \\
\hline
\end{tabular}

Fonte: Dados da Pesquisa (2017)

\subsection{Análise do Teste não Paramétrico: Qui-Quadrado}

Na interpretação do teste de hipótese, ao aplicar o teste do Qui-Quadrado, verifica-se que as frequências observadas apresentam, em relação às frequências esperadas, o nível de significância estatística $(p<0,05)$, quando há diferenças entre as frequências dos grupos pesquisados. 
Considerando a hipótese postulada na aplicação do teste estatístico, pode-se apontar associações significativas entre as variáveis pesquisadas, no qual se deve rejeitar a hipótese H1, para variável 4 (uso de aplicativos para visualizar o trânsito da cidade em tempo real) e para variável 5 (uso de mapas para localizar os pontos turísticos da cidade). Para as demais variáveis (var1, var2 e var3), aceita-se a hipótese $\mathrm{H} 1$, pois não é possível verificar as diferenças estatísticas a respeito das opiniões entre os grupos pesquisados quando se trata do uso de informação para localização de endereços (var1), localização de clientes (var2) e de concorrentes (var3), como pode ser observado no Quadro 3.

Assim, a partir do Quadro 3, foram elaborados de forma resumida os resultados em relação as cinco variáveis analisadas considerando as três categorias de cruzamento, conforme mostrado a seguir: i) sexo; ii) faixa etária; e iii) gênero versus faixa etária.

Quadro 3 - Teste não paramétrico qui quadrado: gênero, faixa etária e gênero/faixa etária

\begin{tabular}{|c|c|c|c|c|c|c|c|}
\hline & VARIÁVEIS - USO DE: & P-valor & $\begin{array}{c}\text { TESTE } \\
\text { HIPÓTESE } \\
\text { GÊNERO }\end{array}$ & P-valor & $\begin{array}{c}\text { Teste } \\
\text { HIPÓTESE } \\
\text { FAIXA ETÁRIA }\end{array}$ & P-valor & $\begin{array}{c}\text { TESTE } \\
\text { HIPÓTESE } \\
\text { GÊNERO/ } \\
\text { FAIXA ETÁRIA }\end{array}$ \\
\hline Var1 & $\begin{array}{l}\text { Mapas como } \\
\text { referência de } \\
\text { localização da } \\
\text { cidade, como bairros, } \\
\text { ruas, etc. (exemplo, } \\
\text { Google Maps) }\end{array}$ & 0,818 & Aceita-se H1 & 0,697 & Aceita-se H1 & 0,300 & Aceita-se H1 \\
\hline Var2 & $\begin{array}{c}\text { Mapas para } \\
\text { localização dos } \\
\text { clientes (exemplo, } \\
\text { Google Maps) }\end{array}$ & 0,153 & Aceita-se H1 & 0,251 & Aceita-se H1 & 0,093 & Aceita-se H1 \\
\hline Var3 & $\begin{array}{l}\text { Mapas para } \\
\text { localização dos } \\
\text { concorrentes } \\
\text { (exemplo, } \\
\text { Google Maps) }\end{array}$ & 0,205 & Aceita-se H1 & 0,165 & Aceita-se H1 & 0,094 & Aceita-se H1 \\
\hline Var4 & $\begin{array}{c}\text { Aplicativos para } \\
\text { visualizar o trânsito } \\
\text { da cidade em tempo } \\
\text { real (exemplo, Waze) }\end{array}$ & 0,232 & Aceita-se H1 & 0,112 & Aceita-se H1 & $0,049 *$ & Rejeita-se H1 \\
\hline Var5 & $\begin{array}{c}\text { Mapas para } \\
\text { localização dos } \\
\text { pontos turísticos da } \\
\text { cidade (exemplo, } \\
\text { Google Maps) }\end{array}$ & $0,048^{*}$ & Rejeita-se H1 & 0,327 & Aceita-se H1 & 0,281 & Aceita-se H1 \\
\hline
\end{tabular}

* Significativo a $5 \%(p<0,05)$

Fonte: Dados da Pesquisa (2017)

Corroborando com os resultados, explica-se os motivos pelos quais as variáveis 4 e 5 devem ser rejeitadas: 
i) Variável 4 (uso de aplicativos para visualizar o trânsito da cidade em tempo real): apresentou um p-valor de 0,049, portanto, menor que 0,05 (5\%) de grau de significância, dessa forma, verificam-se diferenças significativas nas opiniões entre os cidadãos no perfil quanto ao gênero, nesse caso, mais citado por mulheres com menos de 40 anos (90\% de citação).

ii) Variável 5 (uso de mapas para localizar os pontos turísticos da cidade): apresentou um com p-valor de 0,048, portanto, menor que 0,05 (5\%) de grau de significância, assim há diferenças de opiniões, uma vez que as mulheres são as que mais utilizam essa ferramenta, com $83 \%$, enquanto os homens aparecem com $68 \%$.

Quanto aos cruzamentos dos dados obtidos, destacam-se os resultados quanto às opiniões dos entrevistados, que podem ser verificadas a partir do Quadro 4, suas diferenças nas frequências relativas para as variáveis na qual foi rejeitada a hipótese $\mathrm{H} 1$ na var4 por gênero e var5 por gênero/faixa etária.

Quadro 4 - Índice de acesso: gênero, faixa etária e gênero/faixa etária (\%)

\begin{tabular}{|c|c|c|c|c|c|c|c|c|c|c|c|c|c|c|}
\hline & \multicolumn{2}{|c|}{$\begin{array}{l}\text { GÊNERo } \\
\text { FEMININo }\end{array}$} & & \multicolumn{4}{|c|}{ Faixa Etária } & & \multicolumn{4}{|c|}{$\begin{array}{c}\text { AGRUPAMENTO: } \\
\text { GÊNERO X FAIXA ETÁRIA }\end{array}$} & & \\
\hline & Masc. & Fem. & & $20 / 30$ & $31 / 40$ & $41 / 50$ & $50+$ & & $\begin{array}{c}\text { Masc. } \\
\text { Até } \\
40\end{array}$ & $\begin{array}{l}\text { Masc. } \\
40+\end{array}$ & $\begin{array}{c}\text { Fem. } \\
\text { Até } \\
40\end{array}$ & $\begin{array}{l}\text { Fem. } \\
40+\end{array}$ & & Total \\
\hline Var1 & 97,5 & 98,1 & NS & 100,0 & 98,1 & 95,6 & 98,2 & NS & 100,0 & 95,7 & 95,2 & 100,0 & NS & 97,7 \\
\hline Var2 & 72,5 & 82,7 & NS & 77,8 & 84,9 & 71,1 & 69,6 & NS & 82,0 & 65,7 & 85,7 & 80,6 & NS & 75,6 \\
\hline Var3 & 55,0 & 65,4 & NS & 61,1 & 69,8 & 48,9 & 53,6 & NS & 66,0 & 47,1 & 71,4 & 61,3 & NS & 58,1 \\
\hline Var4 & 61,7 & 71,2 & NS & 83,3 & 69,8 & 53,3 & 62,5 & NS & 66,0 & 58,6 & 90,5 & 58,1 & $S^{*}$ & 64,5 \\
\hline Var5 & 68,3 & 82,7 & $S^{*}$ & 55,6 & 77,4 & 71,1 & 75,0 & NS & 68,0 & 68,6 & 81,0 & 83,9 & NS & 72,7 \\
\hline
\end{tabular}

NS: Não significativo e $S^{*}$ : significativo a $5 \%(\mathrm{p}<0,05)$

Fonte: Dados da Pesquisa (2017)

Isso reforça, conforme destacado na literatura, a importância do sistema de informação geográfica, que atribui informações visualizadas por meio de mapas que podem ser codificados por cores os atributos como: renda familiar, gênero, densidade demográfica, entre outros (HESS; RUBIN; WEST, 2003), da mesma forma, nesses serviços que são baseados em mapas, os cidadãos podem clicar em diversos pontos e navegar em todos os lugares desejados para encontrar as localizações desejadas e assim tomar suas decisões conforme planejamento (DING et al., 2003).

\subsection{Análise do Teste não Paramétrico: correlação de Spearman}

Em relação ao teste de correlação de Spearman, ao verificar a hipótese $\mathrm{H} 2$, tem-se a seguinte situação, conforme apontado no Quadro 5, demonstra que há correlação positiva entre todas as variáveis investigadas, entretanto, deve-se rejeitar o teste de hipótese (H2), pois ele não apresenta forte correlação positiva entre elas. 
Quadro 5 - Teste não paramétrico: correlação de Spearman

\begin{tabular}{|c|c|c|c|c|c|}
\hline & VAR1 & VAR2 & VAR3 & VAR4 & VAR5 \\
\hline Var1 & 1 & & & & \\
\hline Var2 & $*, 03$ & 1 & & & \\
\hline Var3 & $*, 09$ & $* *, 59$ & 1 & & \\
\hline Var4 & $*, 17$ & $*, 35$ & $*, 26$ & 1 & 1 \\
\hline Var5 & $*, 13$ & $*, 36$ & $* *, 42$ & $*, 23$ & \\
\hline
\end{tabular}

O símbolo * indica que as correlações verificadas se apresentam da seguinte forma: ** moderada $(0,40$ a 0,69), * fraca (abaixo de 0,39 ).

Fonte: Dados da Pesquisa (2017)

Porém, cabe destacar que as variáveis que apresentaram correlação positiva moderada entre 0,40 a 0,69 foram:

i) A var2 (uso de mapas para localização dos clientes) com a var3 (uso de mapas para localização dos concorrentes) apresentam uma correlação positiva moderada $(0,59)$. Essa correlação positiva moderada entre as variáveis é reforçada por Vlachopoulou et al. (2001), uma vez que ao avaliar por meio de mapas os clientes e concorrentes, reforça que, quanto mais se procura por um endereço de cliente, também aumenta o índice de procura a um endereço de concorrente; esse índice, provavelmente, tem maior implicância quanto ao entrevistado que usa a ferramenta para fins comerciais.

ii) A var4 (uso de aplicativos para visualizar o trânsito da cidade em tempo real, exemplo, Waze) e var5 (uso de mapas para localizar os pontos turísticos da cidade) apresentam correlação positiva moderada $(0,42)$. Também tem uma relação que sugere a seguinte reflexão, pois ao procurar pontos turísticos para uma possível visitação, é de interesse do cidadão procurar o melhor caminho para se chegar ao local e, por sua vez, saber como está o trânsito em tempo real.

\section{Considerações Finais}

Considerando o tema investigado sobre sistema de informação geográfica e geomarketing, verificou-se a importância, os conceitos e a aplicação das ferramentas para apoiar as tomadas de decisões. Assim, referente ao objetivo, que foi analisar o comportamento do cidadão em relação ao uso de informações para tomada de decisão, entende-se que foram respondidas as hipóteses da pesquisa, a qual tinha o propósito de levantar possíveis evidências estatísticas de diferenças entre os grupos abordados, no caso, gênero e faixa etária.

Como principais achados, verificou-se que o uso de mapas digitais (sobretudo o mais conhecido, o Google Maps) é utilizado por $97,7 \%$ dos cidadãos participantes da pesquisa, e ainda que alguns resultados para as variáveis investigadas apontaram diferenças estatísticas entre os perfis dos cidadãos participantes da pesquisa.

O estudo aponta um caminho para uma melhor gestão das cidades com a proposta de qualificar as informações aos cidadãos, uma vez que a conclusão reitera que $98 \%$ dos respondentes usam a ferramenta do Google Maps para localização de endereços, $76 \%$ para localização 
de clientes, $73 \%$ para identificação de pontos turísticos, $65 \%$ para visualização do trânsito em tempo real e $58 \%$ para localização dos concorrentes.

Em resumo, considerando-se as hipóteses postuladas: a $\mathrm{H} 1$, que procurou dimensionar se não há diferença significativa quanto ao uso de mapas virtuais entre os grupos pesquisados (gênero e faixa etária); e H2, que procura estabelecer se há forte correlação positiva entre as variáveis pesquisadas, como achados tem-se que:

Deve-se rejeitar a hipótese $\mathrm{H} 1$ para a variável 4 (uso de aplicativos para visualizar o trânsito da cidade em tempo real) e para a variável 5 (uso de mapas para localizar os pontos turísticos da cidade) e, devido ao apontamento de diferenças de opiniões entre os grupos de cidadãos abordados mediante a aplicação do teste Qui-Quadrado.

Deve-se rejeitar a hipótese $\mathrm{H} 2$ para todas as variáveis, uma vez que não ocorre forte correlação positiva, embora tenha apresentado uma moderada correlação entre as variáveis: a var2 (uso de mapas para localização dos clientes) com a var3 (uso de mapas para localização dos concorrentes); e entre a var4 (uso de aplicativos para visualizar o trânsito da cidade em tempo real, exemplo, Waze) e var5 (uso de mapas para localizar os pontos turísticos da cidade).

Desse modo, entende-se que a pesquisa contribuiu para: i) aprimorar a fundamentação teórica do SIG e geomarketing propiciando um maior esclarecimento para os grupos de pesquisas da área; ii) auxiliar a gestão das cidades considerando os achados dos indicadores quanto ao uso dessas ferramentas; e iii) melhorar a informação e os serviços aos cidadãos como apoio à tomada de decisão.

Como limitação da pesquisa, destaca-se que os resultados não devem ser generalizados, tendo em vista que se trata de uma investigação exploratória mediante uma amostragem não probabilística, o que significa que não expressa necessariamente a mesma realidade de outras cidades de porte semelhante à cidade pesquisada.

\section{Agradecimento}

Agradecemos o Apoio da CNPq Brasil pela Bolsa de Produtividade em Pesquisa do CNPq.

\section{Referências}

AL-HADER, M. et al. Smart city components architicture. In: IEEE. International Conference on Computational Intelligence, Modelling and Simulation. 2009. p. 93-97.

BATCHGEO. Cole seus dados de localização abaixo para mapeá-los. [2017]. Disponível em: https://pt.batchgeo.com/. Acesso em: 31 jul. 2017.

BERNARDO, M.; LIMA, R. da S. Planejamento e implantação de um programa de coleta seletiva: utilização de um sistema de informação geográfica na elaboração das rotas. URBE - Revista Brasileira de Gestão Urbana, [S.I.], v. 9, p. 385-395, 2017.

CALDERONI, L. et al. Location-aware Mobile Services for a Smart City: Design, Implementation and Deployment. Journal of Theoretical and Applied Electronic Commerce Research, Curicó, v. 7 , n. 3, p. 74-87, dez. 2012. 
CASTROGIOVANNI, Antônio Carlos et al. A dialógica entre a Cartografia no ensino básico e o sistema de informação geográfica nos pleitos territoriais. Movimentos para Ensinar Geografia: Oscilações, [S.l.], p. 143-156, 2016.

CAVION, R.; PHILIPS, J. Os Fundamentos do Geomarketing: Cartografia, Geografia e Marketing COBRAC. In: CONGRESSO BRASILEIRO DE CADASTRO TÉCNICO MULTIFINALITÁRIO, Florianópolis, 2006. Anais [...], Florianópolis, 2006.

CERVO, A. L.; BERVIAN, P. A. Metodologia científica. 6. ed. São Paulo: Pearson Prentice Hall, 2007.

CHANG, Y. Spatial cognition in digital cities. International Journal of Architectural Computing, [S.l.], v. 1, n. 4, p. 471-488, 2003.

CORDEIRO, R. A. et al. Uso de Sistema de Informação Geográfica na Gestão do Marketing Mix: o caso de uma rede de calçados femininos. Future Studies Research Journal: Trends and Strategies, [S.I.], v. 9, n. 1, p. 95-114, 2017.

DAMERI, R. P.; COCCHIA, A. Smart city and digital city: twenty years of terminology evolution. In: X CONFERENCE OF THE ITALIAN CHAPTER OF AIS - ITAIS. 2013. Anais [...], 2013. p. 1-8.

DAMERI, R. P. Defining an evaluation framework for digital cities implementation:

International Conference on Information Society (i-Society). [S.I.]: IEEE, 2012.

DAMERI, R. P. et al. Knowledge and Intellectual Capital in Smart City. European Conference on Knowledge Management, Kidmore End, [S.I.], v. 1, p. 250-257, Set. 2014.

DAMERI, R. P.; ROSENTHAL-SABROUX, C. Smart city and value creation. In: SMART CITY. Springer International Publishing. 2014. p. 1-12.

DAMERI, R. P. Searching for Smart City definition: a comprehensive proposal. International Journal of Computers \& Technology, [S.1.], v. 11, n. 5, p. 2.544-2.551, 2013.

DASCALU, O.; ALBULESCU, S. C. Emphasising geomarketing in the automotive industry: dealer network development in romania. International Conference on Management and Industrial Engineering, Bucharest, [S.I.], n. 6, p. 475-480, Nov. 2013.

DING, P. et al. Digital City Shanghai: concepts, foundations, and current state. In: INTERNATIONAL DIGITAL CITIES WORKSHOP. Springer Berlin Heidelberg. 2003. p. 141-165.

FERNANDES, R. T. V. et al. Planejamento urbano "open source": um estudo de caso na identificação de áreas para implantação de aterro sanitário. HOLOS, Natal, v. 33, n. 8, p. 126-144, 2017.

FREITAS, H. et al. O método de pesquisa survey. São Paulo/SP: Revista de administração da USP, São Paulo, v. 35, n. 3, p. 105-112, jul.-set. 2000.

GIL, A. C. Métodos e técnicas de pesquisa social. 6. ed. São Paulo: Atlas, 2008.

HESS, R.; RUBIN, R.; WEST, L. A. Geographic information systems as a marketing information system technology. Decision Support Systems. In Press, Corrected Proof, Available online 8 August 2003.

HIRAMATSU, K.; ISHIDA, T. An augmented web space for digital cities. In: APPLICATIONS AND THE INTERNET. Proceedings: Symposium on. [S.1.]: IEEE, 2001. p. 105-112. 
HOCES, E. et al. Geographic information system supported farm irrigation system design and planning. Water Resources and Irrigation Management, [S.I.], v. 1, n. 1, p. 7-14, Dez. 2012.

HU, Q. Study on Strategy of Tourism Exploitation of Scenic Area with Forested Wetlands based on Geographic Information System. Revista Ibérica de Sistemas e Tecnologias de Informação, Lousada, p. 69-79, Out. 2016.

IBGE - INSTITUTO BRASILEIRO DE GEOGRAFIA E ESTATÍSTICA. Censo 2010. [2011].

Disponível em: mapasinterativos.ibge.gov.br/censo2010. Acesso em: 15 fev. 2017.

ISHIDA, T. Understanding digital cities. In: DIGITAL CITIES. Springer Berlin Heidelberg, 2000. p. 7-17.

ISHIDA, T. Digital city, smart city and beyond. In: PROCEEDINGS OF THE 26TH INTERNATIONAL CONFERENCE ON WORLD WIDE WEB COMPANION. 2017. Anais [...], 2017. p. 1.151-1.152.

KRIŽAN, F. et al. Spatial distribution of consumer preferences: case study of shopping malls in bratislava. Theoretical and Empirical Researches in Urban Management, Bucharest, v. 13, n. 1, p. 13-21, Fev. 2018.

KUMAR, M. R. Digitized Cities and Sustainability: A Study on the role of ICT in development of sustainable cities. Parikalpana: K I I T Journal of Management, Bhubaneswar, v. 12, n. 1, p. 7176, Jan. 2016.

LATOUR, P.; LE FLOC'H, J. Géomarketing: principes, méthodes et applications. Paris: Éditions d'Organisation, 2001.

MACDONALD, K. I. Use and valuation: Information in the city. Urban Studies, London, v. 37, n. 10, p. 1.881-1.892, Set. 2000.

MALHOTRA, K. N. Pesquisa de marketing: uma orientação aplicada. 3. ed. Porto Alegre: Bookman, 2001.

MARCONI, M. A.; LAKATOS, E. M. Fundamentos de metodologia científica. 7. ed. São Paulo: Atlas, 2010.

MARTINS, Gilberto de Andrade. Sobre valide e confiabilidade. Revista Brasileira de Gestão de Negócios, São Paulo, p. 1-12, jan.-abr. 2006.

MATTAR, F. N. Pesquisa de marketing: edição compacta. São Paulo: Atlas, 2000.

REZENDE, D. A. Planejamento de estratégias e informações municipais para cidade digital: guia para projetos em prefeituras e organizações públicas. São Paulo: Atlas, 2012.

ROCHE, S. Geographic Information Science I: Why does a smart city need to be spatially enabled? Progress in Human Geography, London, v. 38, n. 5, p. 703-711, Out. 2014.

ROESCH, S. M. A. Projeto de estágio e de pesquisa em administração: guia para estágios, trabalhos de conclusão, dissertação e estudos de caso. 2. ed. São Paulo: Atlas, 1999.

SCHULER, Doug. Digital cities and digital citizens. In: SCHULER, Doug. Kyoto Workshop on Digital Cities. Springer Berlin Heidelberg, 2001. p. 71-85.

SHAO, Z.; LI, D. Design and implementation of service-oriented spatial information sharing framework in digital city. Geo-Spatial Information Science, [S.l.], v. 12, n. 2, p. 104-109, 2009. 
SIEGEL, S.; CASTELLAN, JR., N. J. Estatística não paramétrica para as ciências do comportamento. São Paulo: Artmed-Bookman, 2017.

SIMÃO, J. B. A concepção de um modelo de cidade digital baseado nas necessidades informacionais do cidadão: o caso dos municípios brasileiros de pequeno porte. Brasília, 2010. 132 f. Tese (Doutorado em Ciência da Informação) - Faculdade de Ciência da Informação, Universidade de Brasília, Brasília, DF, 2010.

VEGA, R. S. et al. Spatial analysis of consumer behavior in a food products market. Theoretical and Empirical. Researches in Urban Management, Bucharest, v. 10, n. 1, p. 25-42, Fev. 2015.

VLACHOPOULOU, M. et al. Geographic information systems in warehouse site selection decisions. International Journal of Production Economics, [S.I.], v. 71, Issues 1-3, 6 May 2001.

W-Q MAO. Study on the Construction and Application of 3D Geographic Information Services for the Smart City (ISPRS). Annals of the Photogrammetry, Remote Sensing and Spatial Information Sciences, Gottingen, v. II, n. 4, p. 41-44, 2014.

XU, H. et al. Research on models of Digital City geo-information sharing platform. In: INTERNATIONAL SYMPOSIUM ON SPATIAL ANALYSIS, SPATIAL-TEMPORAL DATA MODELING, AND DATA MINING, Wuhan, 2009. Anais [...], Wuhan, 2009.

ZHANG, J. et al. A three dimensional modeling and simulation platform design for digital city, Proc. SPIE 5985. In: INTERNATIONAL CONFERENCE ON SPACE INFORMATION TECHNOLOGY, 2016. Anais [...], 2016.

ZHANG, J. et al. Design Research of TIANDITU (Map Worl)-Based Geographic Information System for Travelling Service. The International Archives of Photogrammetry, Remote Sensing and Spatial Information Sciences, Gottingen, v. XL, n. 4, p. 369-372, 2014.

ZHU, X. et al. Metadata-Based Urban Information Management Design. International Journal of e-Education, e-Business, e-Management and e-Learning, Singapore, v. 3, n. 3, p. 274, June, 2013.

\section{Sobre os Autores}

\section{Claudio Luiz Chiusoli}

E-mail: prof.claudio.unicentro@gmail.com

Pós-doutor pelo Programa de Pós-Graduação em Gestão Urbana no Grupo de Pesquisas em Cidade Digital Estratégica.

Endereço profissional: Universidade Estadual do Centro-Oeste (UNICENTRO), Campus Santa Cruz, Departamento de Administração, Rua Salvatore Renna, n. 875, Padre Salvador, Santa Cruz, Guarapuava, PR. CEP: 85015-430.

\section{Denis Alcides Rezende}

E-mail: denis.rezende@pucpr.br

Pós-doutor em Cidade Digital Estratégica (Strategic Digital City) - DePaul University - School of Public Service, Chicago,USA.

Endereço profissional: Pontifícia Universidade Católica do Paraná (PUCPR), Programa de Pós-Graduação em Gestão Urbana, Rua Imaculada Conceição, n. 1.155, Bloco 2, Prado Velho, Curitiba, PR. CEP: 80215-901. 